\title{
Assessment of Genetic Diversity of Some Finger Millet (Eleusine coracana (L.) Gaertn. Accessions Using Morphological Markers
}

\author{
D.V.S. Kaluthanthri ${ }^{1}{ }^{*}$ and P.N. Dasanayaka ${ }^{1}$ \\ ${ }^{1}$ Department of Botany, University of Sri Jayewardenepura, Nugegoda, Sri Lanka
}

Date Received: 16-11-2016

Date Accepted: 22-12-2016

\begin{abstract}
Germplasm characterization is an important link between conservation and utilization of plant genetic resources. The study was conducted to characterize randomly selected 20 finger millet germplasm accessions obtained from Plant Genetic Resource Center, Gannoruwa, Sri Lanka using morphological markers. Morphological study was carried out using Randomized Complete Block Design (RCBD) and 15 morphological markers were recorded. Analysis of variance (ANOVA) results for quantitative morphological characters revealed that all quantitative morphological characters measured differed significantly $(p<0.05)$ among the accessions used for the study, indicating higher levels of morphological diversity. According to the ANOVA results, days to flowering and days to maturity show high level of predictive capability while flag leaf length and number of productive tillers show comparatively low level of predictive capability. Principal component analysis indicated that morphological characters such as days to flowering, finger number and yield per plant were the important traits contributing for the overall variability implying that breeding effort on those traits can meet the targeted objective. The clustering pattern of studied finger millet accessions based on morphological markers comprised of two major clusters. Both clusters comprised of Indian accessions those conserved at PGRC, Gannoruwa and as well as Sri Lankan accessions.
\end{abstract}

Results of the study suggest a considerable morphological variability, which could exist among the studied traits. Furthermore, this study revealed that the genetic diversity existed irrespective to the geographical origin. This finding justifies the importance of germplasm characterization.

Keywords: Finger Millet, Morphological Markers, Germplasm Accessions, Genetic Diversity, Crop Improvement

\section{Introduction}

Finger millet, E. coracana (L.) Gaertn. is a tetraploid crop $(2 n=4 x=36)$ belonging to the order Poales, family Poaceae, sub family Chloridoideae.

This crop is widely cultivated in tropical and semi tropical regions of the world including Africa, India, China, Japan, Australia and Sri Lanka as well. E. coracana has a greater nutritional value. Both grain and hull of finger millet have considerable nutrient content. The grain contains carbohydrate, protein, fat, fibers, iron, calcium, minerals and essential amino acids such as leucine, tryptophan, phenylalanine and methionine. The presence of methionine is very important for the people who are depending on the staple

*Correspondence: nilanthiedas@sjp.ac.lk

Tel: +94718122358

ISSN 2235-9370 Print/ISSN 2235-9362 Online (C) University of Sri Jayewardenepura 
foods that lack methionine such as cassava, maize and polished rice. On the other hand finger millet has considerably higher amount of calcium compared to other cereals. The hull contains protein, fiber, calcium and phosphorus. Consequently, this can be considered as the major preventive agent against malnutrition. Content of each component can be changed according to the crop variety. However each and every variety has its own nutritive value. Finger millet has the ability to produce higher yield than other crops under multiple stresses such as drought, soil acidity and land marginality (Babu and Hilu, 1993; Upadgyaya et al., 2006). Moreover it has excellent storage qualities (Dida et al., 2007)

Germplasm characterization is an important link between conservation and utilization of plant genetic resources in crop improvement programmes. There are more than 200 accessions of finger millet conserved at Plant Genetic Resource Center (PGRC), Gannoruwa, Sri Lanka. Understanding of genetic diversity of those conserved accessions is an important to use them in crop improvement programmes. Among the multivariate techniques, principal component analysis (PCA) and cluster analysis had been shown to be very useful in selecting genotypes for breeding program that meet the objective of a plant breeder (Mohammadi and Prasanna, 2003). PCA may be used to reveal patterns and eliminate redundancy in data sets (Adams, 1995). Upadhyaya et al., 2007, Ulaganathan and Nirmalakumari; 2015, Dagnachew et al., 2012 and Goswami et al., 2015 have been used morphological markers for the characterization of finger millet germplasm accessions. The study was conducted to assess phenotypic diversity of randomly selected 20 finger millet germplasm accessions conserved at PGRC, Gannoruwa, Sri Lanka. The important objective of any plant scientist is to identify an optimum number of plant traits which are sufficient to explain the maximum variability in the crop growth from sowing to harvest (Ulaganathan and Nirmalakumari, 2015).

\section{Materials and Methods}

\subsection{Plant Material}

A total of randomly selected 20 finger millet (Eleusine corocana subsp. Corocana) germplasm accessions were used in this study (Table 01). Seeds of these 20 accessions were obtained from the seed gene bank of Plant Genetic Resources Centre (PGRC), Gannoruwa, Sri Lanka.

\subsection{Morphological Characterization \\ Experimental Design}

Seeds of each accession were sown in small plastic pots filled with normal soil. Pots were irrigated soon after sowing and placed in the plant house for germination. Twenty days old seedlings were transplanted separately in to the large plastic pots filled with mixture of soil, sand and compost (1:1:1) as each pot contained 4 plants. A total of 20 accessions were arranged in a Randomized Complete Block Design (RCBD) with 12 individuals from each accession.

\section{Data Collection}

Quantitative traits were recorded at different growth stages of the crop including flowering stage, dough stage, maturity stage and post-harvest stage from all plants (Table 2). Traits were scored following International Plant genetic Resource Institute (IPGRI) descriptors developed for finger millet. 
Table 1: Details of the finger millet germplasm accessions used for the study.

\begin{tabular}{lclll}
\hline \multicolumn{1}{c}{ Accession } & Accession No. & Accession Name & Origin of Accession & Organization \\
\hline 1/ GalB-Ham & 000190 & Gal Bora & Hambanthota & PGRC \\
2/ GPU5-Ind & 000928 & GPU 5 & India & Unknown \\
3/ PES110-Ind & 000927 & PES 110 & India & Unknown \\
4/ BalaK-Rat & 001331 & Bala Kurakkan & Rathnapura & PGRC \\
5/ GPU114-Ind & 000926 & GPU 114 & India & Unknown \\
6/ KM1-Ind & 000964 & KM - 1 & India & RARS/AK \\
7/ EdalK-Ham & 000192 & EDAL - KUR & Hambanthota & PGRC \\
8/ PR202-Ind & 000925 & PR 202 & India & Unknown \\
9/ BalaK-Kan & 001815 & Bala Kurakkan & Kandy & PGRC \\
10/ INDAE9-Ind & 000909 & INDAE/9 & India & Unknown \\
11/ JNR3B-Ind & 000962 & JNR - 3B - 1008 & India & RARS/AK \\
12/ Kur-N.eli & 000960 & Kurakkan & Nuwara Eliya & PGRC \\
13/ SEL4-Ind & 000911 & SEL No - 4 & India & Unknown \\
14/ EdalK-Kur & 000426 & EDAL Kurakkan & Kurunegala & PGRC \\
15/ GalK-Kur & 000418 & GAL Kurakkan & Kurunegala & PGRC \\
16/ KiriMK-Rat & 001304 & KiriMora Kurakkan & Rathnapura & PGRC \\
17/ KaluGK-Kan & 001828 & Kalu Gal Kurakkan & Kandy & PGRC \\
18/ Kur-Anu & 001201 & Kurakkan & Anuradhapura & PGRC \\
19/ HR374-Ind & 000910 & HR 374 & India & PGRC \\
20/ KaluGK-Mat & 001233 & Kalu Gal Kurakkan & Matale & PGRC \\
\hline
\end{tabular}

\subsection{Statistical Analysis}

Principal Component Analysis

Principal component analysis for 14 quantitative traits was performed using MINITAB14 software. As suggested by Johnson and Wichern (1988) principal components with eigen values greater than one was considered.

\section{Cluster Analysis}

Hierarchical clustering of complete linkage method with squared Euclidian distance was performed using MINITAB14 software. Data of all quantitative traits were standardized to a mean of zero and a variance of one before clustering to avoid bias that arise due to differences in measurement scales.

Table 2: Descriptors used in morphological characterization of finger millet accessions.

\begin{tabular}{cc}
\hline & Morphological Descriptors \\
\hline Flowering Stage & Maturity Stage \\
Days to flowering & Number of productive tillers \\
Flag leaf length $(\mathrm{mm})$ & Days to maturity \\
Flag leaf width $(\mathrm{mm})$ & Culm branching
\end{tabular}




\section{Dough Stage}

Plant height $(\mathrm{cm})$

Culm thickness (mm)

Finger number

Finger length (mm)

Finger width $(\mathrm{mm})$

\section{Post Harvest Stage}

Weight of sun dried ear at maturity $(\mathrm{g})$

Weight of grains per ear at maturity $(\mathrm{g})$

1000 grain weight $(\mathrm{g})$

Grain yield per plant $(\mathrm{g})$

\section{Results}

\subsection{Morphological characterization}

Quantitative morphological data were analyzed using software MINITAB version 14 to derive Descriptive statistics, Analysis of variance and multivariate discrimination of quantitative morphological characters. There were fifteen quantitative morphological characters all together. They were days to flowering, flag leaf length, flag leaf width, plant height, culm thickness, finger number, finger length, finger width, number of productive tillers, days to maturity, weight of sun dried ear, weight of grains per ear, 1000 grain weight, yield per plant and culm branching. Out of these characters culm branching was a monomorphic character.

\section{Analysis of variance (ANOVA) of quantitative morphological markers}

ANOVA Results for quantitative morphological characters revealed that all quantitative morphological characters measured differed significantly $(p<0.05)$ among the accessions used for the study, indicating higher levels of morphological diversity (Table 3 ). According to the ANOVA results, days to flowering $\left(\mathrm{F}=37.55\right.$, adj- $\left.\mathrm{R}^{2}=75.77 \%\right)$ and days to maturity $\left(\mathrm{F}=51.66\right.$, adj- $\left.\mathrm{R}^{2}=81.6 \%\right)$ show high level of predictive capability while flag leaf length $\left(\mathrm{F}=4.3\right.$, adj- $\left.\mathrm{R}^{2}=22.27 \%\right)$ and number of productive tillers $\left(\mathrm{F}=3.99\right.$, adj- $\left.\mathrm{R}^{2}=20.77 \%\right)$ show comparatively low level of predictive capability.

Table 3: Results of Analysis of variance with respect to twenty accessions.

\begin{tabular}{lccc}
\hline $\begin{array}{c}\text { Quantitative morphological } \\
\text { character }\end{array}$ & F value & $\begin{array}{c}\text { P value (Observed } \\
\text { significant value) }\end{array}$ & $\begin{array}{c}\text { Adj-R }{ }^{2} \text { value (Adjusted } \\
\text { coefficient of) }\end{array}$ \\
\hline Days to flowering & 37.55 & 0.00 & $75.77 \%$ \\
Flag leaf length & 4.30 & 0.00 & $22.27 \%$ \\
Flag leaf width & 6.67 & 0.00 & $32.96 \%$ \\
Plant height & 7.78 & 0.00 & $37.05 \%$ \\
Culm thickness & 11.83 & 0.00 & $48.45 \%$ \\
Finger number & 11.55 & 0.00 & $47.91 \%$ \\
Finger length & 8.19 & 0.00 & $38.51 \%$ \\
Finger width & 10.67 & 0.00 & $45.73 \%$ \\
No of productive tillers & 3.99 & 0.00 & $20.77 \%$ \\
Days to maturity & 51.66 & 0.00 & $81.60 \%$ \\
Weight of sun dried ear & 6.08 & 0.00 & $33.71 \%$ \\
Weight of grains per ear & 6.14 & 0.00 & $33.96 \%$ \\
1000 grain weight & 7.33 & 0.00 & $38.77 \%$ \\
Yield per plant & 5.73 & 0.00 & $32.09 \%$ \\
\hline
\end{tabular}

\section{Principal Component Analysis (PCA)}

Principal component analysis (PCA) was performed for all measured quantitative morphological characters. The first four principal components having eigen values greater than one were extracted from the mean of fourteen normalized quantitative traits of 20 finger millet accessions. A variance of $49.0 \%, 13.4 \%, 12.4 \%$ and $8.4 \%$ were extracted from the first to the fourth components respectively. More than $90 \%$ variation was extracted from the first 
six principal components (Table 4). As shown in Table 5, morphological characters such as days to flowering, finger number, weight of sun dried ear, weight of grains per ear, 1000 grain weight and yield per plant were the major contributors for the variation observed in the first principal component. The variation in the second principal component was mainly due to flag leaf length, number of productive tillers and culm thickness. Likewise, flag leaf length and finger length were the major contributors to the variation in the third principal component. The variability in the fourth component was attributed mainly due to flag leaf width and culm thickness. Data from first two components were used to derive PCA score plot and loading plot (figure 1 and 2). The studied 20 accessions were divided into four groups (A, B, C and D) based on the first two principal components as shown in PCA score plot (figure 1). Similarly, the recorded traits were divided into four groups depending on the first two principal components as shown in PCA loading plot (figure 2). Days to flowering and days to maturity were mainly responsible for the morphological diversity existed in group B accessions (6/KM1-Ind, 13/SEL4-Ind, 14/EdalK-Kur, 15/GalK-Kur, 16/KiriMK-Rat, 17/KaluGK-Kan, 18/Kur-Anu and 20/KaluGK-Mat) and as flag leaf length, culm thickness and number of productive tillers were responsible for the morphological diversity existed in group C accessions (8/PR202-Ind, 10/INDAE9-Ind and 19/HR374-Ind).

Table 4: Principal components showing the Eigen values, proportion of variation and total variation across axis.

\begin{tabular}{cccc}
\hline Principal & Eigen value & Proportion of & Total variation \\
\hline 1 & 6.8567 & 49.0 & 0.490 \\
2 & 1.8777 & 13.4 & 0.624 \\
3 & 1.7365 & 12.4 & 0.748 \\
4 & 1.1805 & 8.4 & 0.832 \\
5 & 0.6177 & 4.4 & 0.876 \\
6 & 0.5077 & 3.6 & 0.913 \\
7 & 0.4588 & 3.3 & 0.945 \\
8 & 0.3315 & 2.4 & 0.969 \\
9 & 0.1675 & 1.2 & 0.981 \\
10 & 0.1191 & 0.9 & 0.990 \\
11 & 0.0916 & 0.7 & 0.996 \\
12 & 0.0489 & 0.3 & 1.000 \\
13 & 0.0051 & 0.0 & 1.000 \\
14 & 0.0007 & 0.0 & 1.000 \\
\hline
\end{tabular}




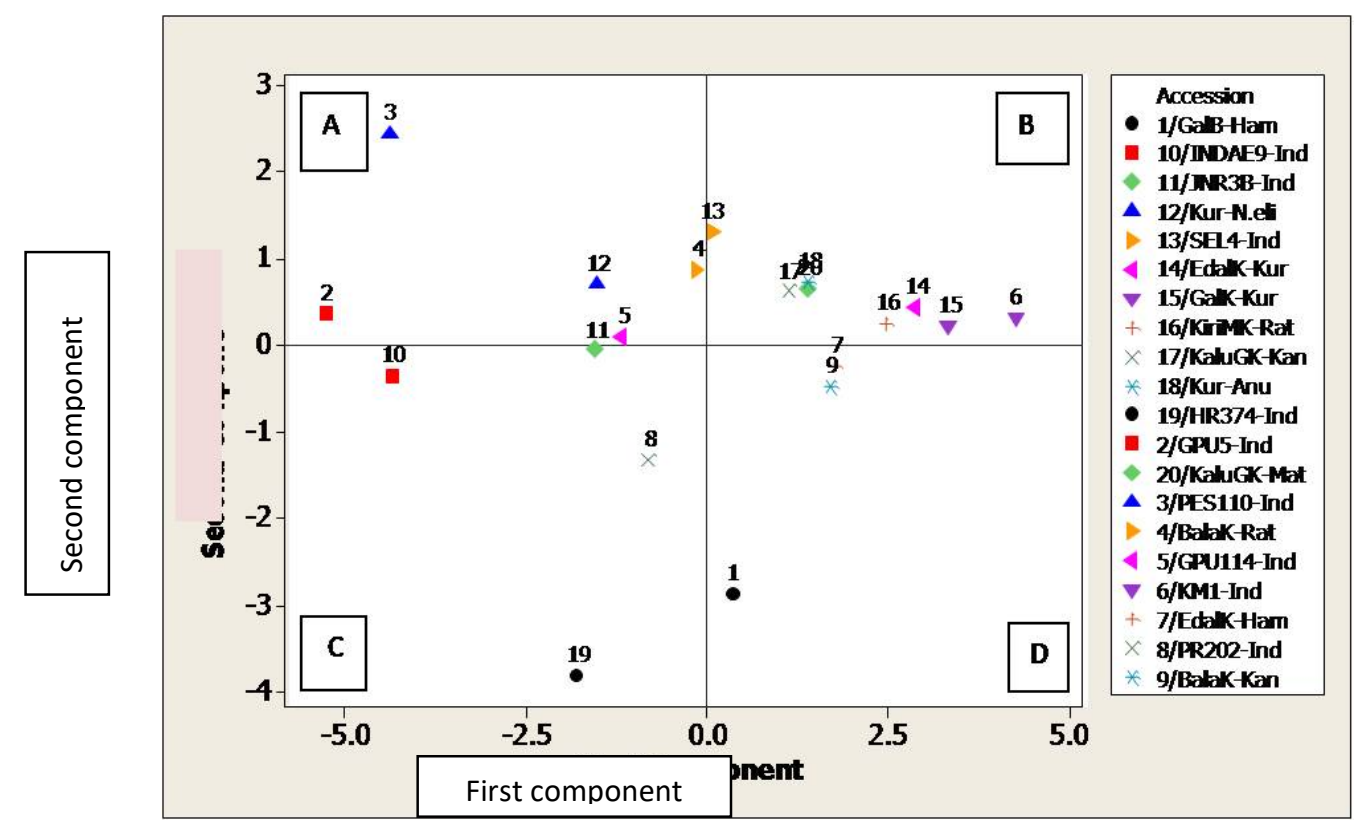

Figure1: PCA score plot for twenty finger millet germplasm accessions.

Table 5: Principal component analysis for 14 quantitative traits in 20 finger millet accessions - non- rotated loadings.

\begin{tabular}{lcccccc}
\hline \multicolumn{1}{c}{ Character } & PC1 & PC2 & PC3 & PC4 & PC5 & PC6 \\
\hline Days to flowering & 0.329 & 0.112 & -0.171 & -0.177 & -0.361 & 0.158 \\
Flag leaf length (mm) & -0.054 & -0.360 & -0.555 & -0.149 & 0.063 & -0.154 \\
Flag leaf width (mm) & -0.215 & 0.293 & 0.060 & -0.532 & 0.038 & -0.270 \\
Plant height (cm) & -0.215 & 0.272 & -0.369 & -0.022 & 0.494 & 0.365 \\
Culm thickness (mm) & -0.116 & -0.456 & 0.116 & -0.555 & 0.320 & 0.167 \\
Finger number & -0.328 & -0.065 & 0.172 & -0.270 & -0.161 & 0.113 \\
Finger length (mm) & -0.255 & 0.016 & 0.430 & 0.139 & 0.163 & 0.457 \\
Finger width (mm) & -0.241 & 0.181 & -0.374 & 0.220 & 0.298 & -0.182 \\
No of productive tillers & -0.035 & -0.607 & -0.151 & 0.263 & -0.096 & 0.257 \\
Days to maturity & 0.280 & 0.274 & -0.180 & -0.099 & -0.041 & 0.593 \\
Weight of sun dried ear $(\mathrm{g})$ & -0.351 & 0.071 & -0.109 & -0.103 & -0.371 & 0.082 \\
Weight of grains per ear $(\mathrm{g})$ & -0.359 & 0.064 & -0.139 & 0.027 & -0.320 & 0.062 \\
1000 grain weight $(\mathrm{g})$ & -0.313 & 0.020 & 0.188 & 0.350 & 0.077 & -0.112 \\
Yield per plant $(\mathrm{g})$ & -0.351 & 0.015 & -0.184 & 0.038 & -0.346 & 0.147 \\
\hline
\end{tabular}




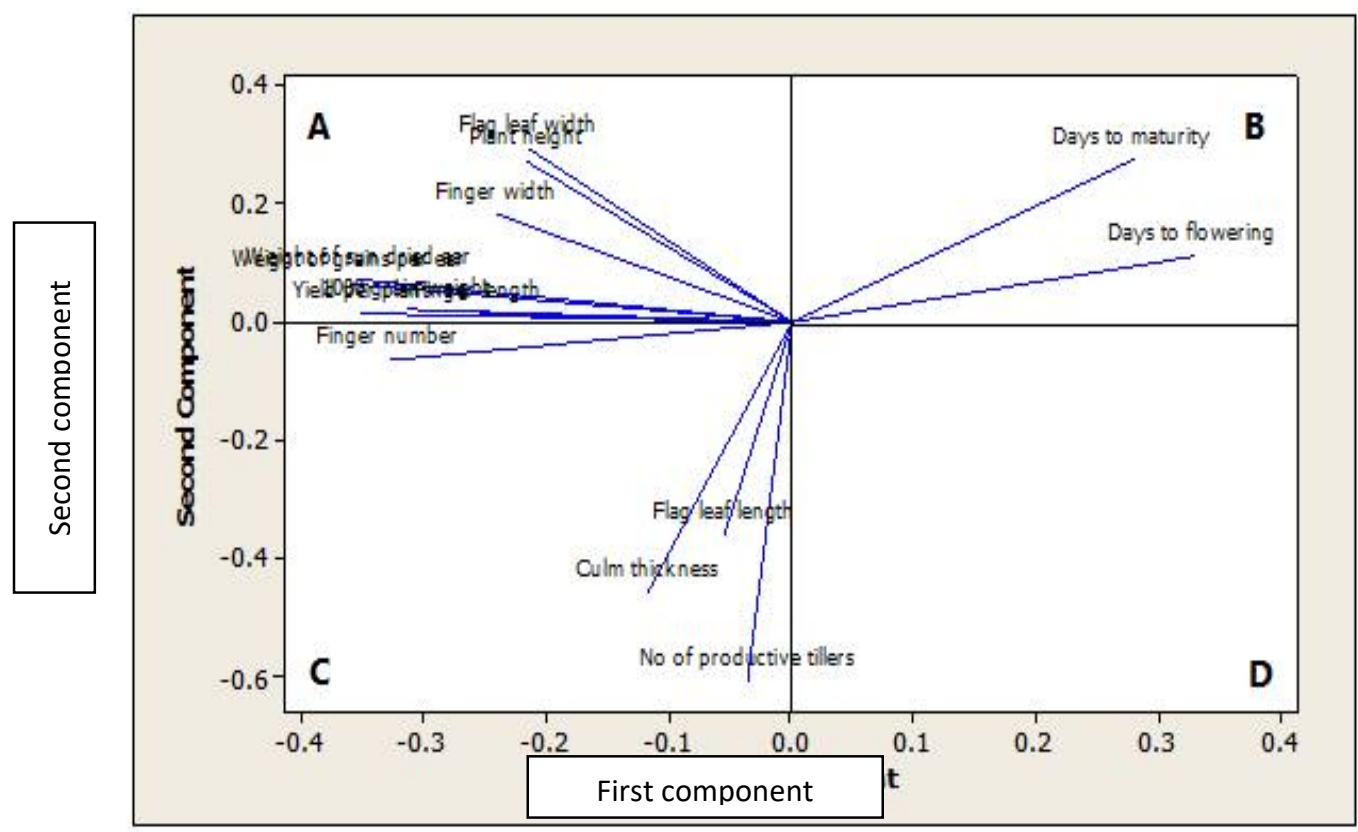

Figure 2: PCA loading plot for fourteen quantitative traits in finger millet accession.

\section{Cluster Analysis}

Cluster analysis was performed for all multivariate data derived from quantitative morphological characters with respect to each individual. The clustering pattern of finger millet accessions based on morphological markers is depicted in the figure 3 . The dendrogram constructed by morphological markers comprises two major clusters as cluster 1 and cluster 2 (figure 3). Ten accessions have grouped into cluster 1 with about $40 \%$ of similarity while the rest of ten accessions have grouped into cluster 2 with about $50 \%$ of similarity. 


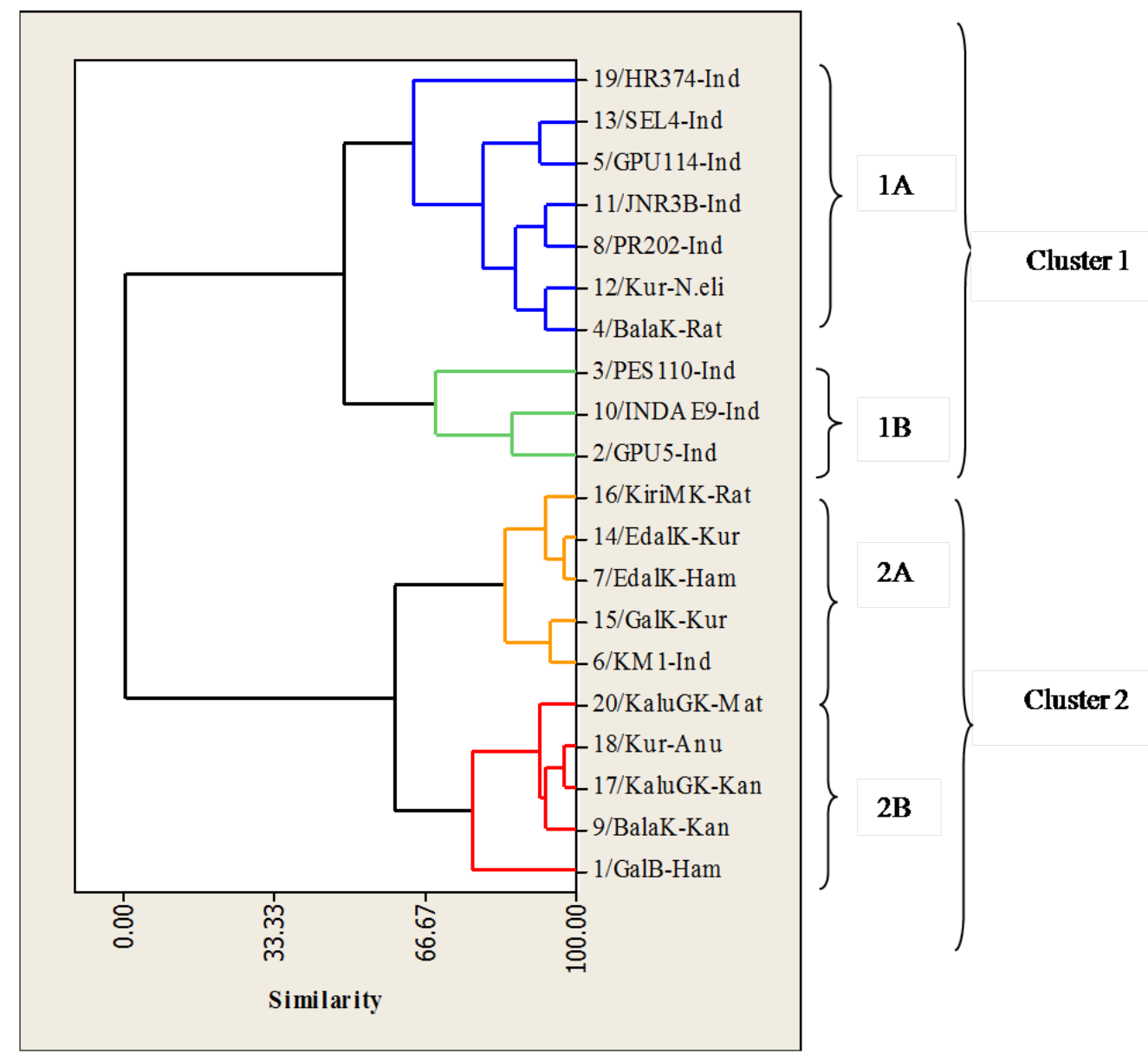

Figure 3: Dendrogram of twenty finger millet germplasm accessions based on Squared Euclidean distances.

Cluster 1 has two sub-clusters as sub-cluster 1A and sub cluster 1B. Sub-cluster 1A comprised of five Indian accessions (19/HR374-Ind, 13/SEL4-Ind, 5/GPU114-Ind, 11/JNR3B-Ind and 8/PR202-Ind) and two Sri Lankan accessions (4/BalaK-Rat [accession from Rathnapura] and 12/Kur-N.eli [accession from Nuwara Eliya]). Sub-cluster 1B has three Indian accessions (3/PES110-Ind, 10/INDAE and 2/GPU5-Ind). Within the sub-cluster 1A, three mini-clusters can be identified. In here, 13/SEL4-Ind (accession from India) and 5/GPU114-Ind (accession from India) grouped into one of mini-clusters. 8/PR202-Ind (accession from India) and 11/JNR3B-Ind (accession from India) grouped into one minicluster and 12/Kur-N.eli (accession from Nuwara Eliya) and 4/BalaK-Rat (accession from Rathnapura) grouped into the other mini-cluster with low level of genetic differences between each other. Apart from these three mini-clusters, 19/HR374-Ind (accession from India) formed a separate mini-cluster individually. Within the sub cluster 1B, 10/INDAE9-Ind (accession from India) and 2/GPU5-Ind (accession from India) grouped into a mini-cluster while 3/PES110-Ind (accession from India) formed a separate mini cluster individually. Cluster 2 has two-sub clusters as sub-cluster 2A and sub-cluster 2B. Sub-cluster 2A comprised of one Indian accession (6/KM1-Ind) and four Sri Lanka accessions (16/KiriMKRat [accession from Rathnapura], 14/EdalK-Kur [accession from Kurunegala], 7/EdalK-Ham [accession from Hambanthota] and 15/GalK-Kur [accession from Kurunegala]). Sub-cluster 2B comprised of five of Sri Lankan accessions (20/KaluGK-Mat [accession from Matale], 18/Kur-Anu [accession from Anuradhapura], 17/KaluGK-Kan [accession from Kandy], 
9/BalaK-Kan [accession from Kandy] and 1/GalB-Ham [accession from Hambanthota]). Within sub-cluster 2A, two mini-clusters can be identified. 16/KiriMK-Rat (accession from Rathnapura), 14/EdalK-Kur (accession from Kurunegala) and 7/EdalK-Ham (accession from Hambanthota) grouped into one of mini-clusters while 15/GalK-Kur (accession from Kurunegala) and 6/KM1-Ind (accession from India) grouped into another mini-cluster with low level of genetic differences with each other. Within the sub-cluster 2B, 20/KaluGK-Mat (accession from Mathale), 18/Kur-Anu (accession from Anuradhapura), 17/KaluGK-Kan (accession from Kandy) and 9/BalaK-Kan (accession from Kandy) grouped into a minicluster with low level of genetic differences with each other while 1/GalB-Ham (accession from Hambanthota) formed a separate mini-cluster individually (Figure 3).

\section{Discussion}

The analysis of variance showed highly significant differences among the accessions for all studied quantitative morphological characters. This revealed that the studied accessions were genetically diverse and the significant amount of variability existed in the plant material. Therefore, there is an opportunity to undertake further breeding activities like hybridization program. This is supported by the substantial variations in finger millet that have been reported in previous studies by Naik et al., (1994) and Prasado et al., (1994).

Maximum positive correlation observed for most of the characters with grain yield per plant revealed that all these characters could be simultaneously improved and it also suggested that increase in any one of them would lead to improvement of yield per plant. This further reveals the fact that the yield per plant is a character which is influenced by numerous other characters. Therefore knowledge about the association between yield per plant and other characters helps in improving the selection efficiency in breeding programs. The correlation between characters may exist due to several factors including both genetic and epigenetic influences. In this case, pleiotropy and genetic linkage play major roles.

According to the ANOVA results, days to flowering and days to maturity showed high level of predictive capability while flag leaf length and number of productive tillers showed comparatively low level of predictive capability. Early maturing genotypes have a great advantage in cases where terminal drought is common occurrence. It also escapes high pest or disease incidence, abnormal weather conditions and field is timely vacated for sowing of next crop. Days to maturity is very important from the view of harvesting of the produce since finger millet has dual advantage of grain as well as fodder (Sumathi et al., 2007). Satish, 2003 and Sharthabu, 2005 have reported that length of the finger has direct association with grain yield of finger millet.

The principal component analysis is a statistical way of identifying plant traits which have major contributions to the observed variation among the studied accessions. Results of this analysis can be used in the selection of suitable accessions with particular traits for breeding purpose. Principal component analysis in this study confirmed the first principal component contributed maximum number of characters towards genetic diversity and those traits could be effectively used for further breeding programs to create more variability. Days to flowering, flag leaf width, plant height, culm thickness, finger number, finger length, finger width, days to maturity, weight of sun dried ear, weight of grains per ear, 1000 grain weight and yield per plant were the most important traits contributing to the overall variability observed among the accessions. Characters with high variability are very important in breeding programs. According to the cluster analysis for quantitative 
morphological traits, 20 accessions were separated into two main clusters based on Squared Euclidean distances and complete linkage method.

Out of studied accessions, individuals of 19-HR374-Ind accession produced the highest number of ears per plants, but the sizes of the ears were very small in most cases. Therefore, the yield per plant from such individuals was not significantly very high.

However, potential environmental influence on the phenotypes makes the process of evaluation more complex and difficult because phenotypic variations occurring due to changed in environmental conditions which could be erroneously scored in the process of germplasm characterization thus one should be careful with false positives and false negatives when the environment affects specific morphotypes (Ferreria, 2005).

In the present study, both local and exotic accessions have been studied and studied germplasm accessions could not be differentiated into distinct groups as local and exotic depending on morphological relatedness among the accessions. Therefore, it revealed that the genetic diversity does not depend on the geographical origin. Germplasm accessions collected from same district showed considerable genetic distance whereas germplasm accessions collected from different districts showed considerable genetic similarity.

\section{Conclusion}

Development of new varieties, collection of genetic resource and conservation of genetic resources depend on the presence of specific character or characters. Evaluation and characterization of accessions with respect to such characters are important for accomplishing varietal development, genetic resource collection and conservation. In that case, results of multivariate analysis provide a way for estimating morphological diversity among accessions. Those results are useful for the evaluation of potential breeding values of studied accessions. Findings of this study suggest the requirement for breeders to exploit accession from distinct groupings for the development of improved varieties. There was a significant genetic variation for all quantitative morphological traits among the accessions used in this study according to the principal component analysis. Furthermore, this study revealed that the genetic diversity existed irrespective to the geographical origin. This finding justifies the importance of germplasm characterization.

\section{References}

Adams, M.W., 1995. An Estimate of Homogeneity in Crop Plants with Special Reference to Genetic Vulnerability in Dry Season. Phseolus vulgaris. Euphytica, 26: 665-679.

Babu, W.E. and Hilu, K.W., 1993. Protein, Calcium, Iron and Amino Acid Content of Selected Wild and Domesticated Cultivars Of Finger Millet. Plant Foods Hum. Nutr. 43: 97-104.

Dagnachew, L., Kassahun, T., Masresha, F. and Santie, D.V., 2012. Multivariate Analysis for Quantitative Traits in Finger Millet (Eleusine coracana Subsp. coracana) Population Collected from Eastern and South-eastern Africa: Detection for Patterns of Genetic Diversity. Int. J. Agric. Res. 7(6): 303-314.

Dida, M.M., Srinivasachary, S., Ramakrishnan, J.L., Bennetzen, M.D. and Devos, K.M., 2007. The Genetic Map of Finger Millet, Eleusine coracana. Theor. Applied Genet. 114:321-332. 
Ferreira, M.E., 2005. Molecular Analysis of Gene Banks for Sustainable Conservation and Increased Use of Crop Genetic Resources. The Role of Biotechnology. Villa Gualino, Turin, Italy, 5-7.

Goswami, A.P., Prasad, B. and Joshi, V.C., 2015. Characterization of Finger Millet [Eleusine coracana (L.) Gaertn.] Germplasm for Morphological Parameters under Field Conditions. Journal of Applied and Natural Science 7 (2) : 836 - 838.

Johnson, R.A. and Wichern, D.W., 1988. Applied Multivariate Statistical Analysis. PrenticaHill: Englewood chiffs, NJ.

Mohammadi, S.A. and Prasanna, B.M., 2003. Analysis of Genetic Diversity in Crop PlantsSalient Statistical Tools and Considerations. Crop Sci. 43:1235-1248.

Naik, B.J., Shankare, B.T.G. and Seetharam, A., 1994. Pattern of Variability in Relation to Domestication of Finger Millet in Africa and India. In K.E. Riley., 347-363.

Prasado, K.E.R., Wet, D.J.M.J., Reddy, G. V. and Mengesha, M.H., 1994. Diversity in the Small Millets Collection at ICRISAT. In K.E. Riley, .331-345.

Satish, D., 2003, Studies on Genetic Diversity Based on Productive and Variability for Quality Traits in Finger Millet (Eleusine coracana Gaertn.). M.Sc. (Agri.) Thesis, Univ. Agric. Sci., Dharwad.

Sharthabu K. S., 2005, Stability Analysis in White Ragi (E. coracana Gaertn.) Genotypes. M.Sc. (Agri) Thesis, Univ. Agric., Dharwad.

Sumathi, P., John Jeol, A., and Muralidharan, V., 2007, Genetic Variability in the Hybrids of Finger Millet (E. coracana (L.) Gaertn.). J. Crop Res., 33 (1,2 and 3): 192-194.

Ulaganathan, V. and Nirmalakumari, A., 2015. Finger Millet Germplasm Characterization and Evaluation Using Principal Component Analysis. SABRAO Journal of Breeding and Genetics. 47 (2), 79-88.

Upadhyaya, H.D., Gowda, C.L.L., Pundir, R.P.S., Reddy, V.G. and Singh, S., 2006. Development of Core Subset of Finger Millet Germpllasm Using Geographical Origin and Data on 14 Quantitative Traits. Gen. Res. Crop Evo. 53:679-685.

Upadhyaya, H. D., Gowda, C. L. L., Gopal Reddy, V., 2007. Morphological Diversity in Finger Millet Germplasm Introduced from Southern and Eastern Africa. SAT eJournal, J. SAT Agric. Res.,

http://www.icrisat.org/journal/Sorgum_Millet_other_Cereals3.htm Accessed $2^{\text {nd }}$ November, 2015 . 